\title{
The dilemma of aspirin resistance in obese patients
}

\author{
Devarshi Ardeshna ${ }^{1}$, Sarthak Khare ${ }^{2}$, Pooja S. Jagadish ${ }^{3}$, Venugopal Bhattad ${ }^{4}$, Brandon Cave ${ }^{5}$, \\ Rami N. Khouzam ${ }^{6}$
}

${ }^{1}$ College of Medicine, University of Tennessee Health Science Center, Memphis, TN, USA; ${ }^{2}$ Department of Medicine, St Elizabeth's Medical Center, Tufts University School of Medicine, Boston, MA, USA; ${ }^{3}$ Department of Internal Medicine, University of Tennessee Health Science Center, Memphis, TN, USA; ${ }^{4}$ Department of Cardiovascular Diseases, East Tennessee State University, Johnson City, TN, USA; ${ }^{5}$ Department of Pharmacy, Methodist University Hospital, Memphis, TN, USA; ${ }^{6}$ Division of Cardiovascular Diseases, Department of Internal Medicine, University of Tennessee Health Science Center, Memphis, TN, USA

Contributions: (I) Conception and design: D Ardeshna, S Khare, P Jagadish, V Bhattad; (II) Administrative support: R Khouzam; (III) Provision of study materials or patients: All authors; (IV) Collection and assembly of data: All authors; (V) Data analysis and interpretation: All authors; (VI) Manuscript writing: All authors; (VII) Final approval of manuscript: All authors.

Correspondence to: Devarshi Ardeshna. College of Medicine, University of Tennessee Health Science Center, 910 Madison Ave. \#1002, Memphis, TN 38103, USA. Email: dardesh2@uthsc.edu.

\begin{abstract}
Aspirin resistance (AR) commonly refers to the concept of reduced aspirin efficacy in preventing cardiovascular disease and platelet inhibition. Obesity increases the risk of heart disease three- to fourfold and has been associated with AR. Aspirin is used as a tool for both primary and secondary prevention, but recent studies suggest that its lack of efficacy for primary prevention is partly attributable to obesity. Several mechanisms have been described that contribute to AR in obese patients using pharmacokinetics and pharmacodynamics. AR may be attenuated through weight loss, alternative dosing regimens, and different drug formulations. With the global rise of obesity, it is imperative to find preventive therapies that adequately address atherosclerotic cardiovascular disease (ASCVD) risk in this population.
\end{abstract}

Keywords: Aspirin resistance (AR); obesity; aspirin

Submitted Jun 25, 2019. Accepted for publication Jul 15, 2019.

doi: $10.21037 /$ atm.2019.07.52

View this article at: http://dx.doi.org/10.21037/atm.2019.07.52

\section{Introduction}

Aspirin has long been considered a stalwart for the prevention of atherosclerotic cardiovascular disease (ASCVD) due to its prominent antiplatelet effects (1). While the benefit of aspirin in secondary prevention remains salient $(1,2)$, more recent studies have called into question the role of aspirin in the primary prevention of ASCVD (3-5). In fact, the American Association of Family Physicians, American Heart Association, and American College of Cardiology have all recently changed their guidelines to recommend against aspirin use for primary prevention because the bleeding risk outweighs the net clinical benefit of decreased ASCVD events $(1,6)$. While numerous studies performed in 1990s showed that aspirin is beneficial in the primary prevention of cardiovascular event $(2,7)$, more recent studies have failed to demonstrate as profound of a benefit, especially with bleeding risk is considered $(8,9)$. One theory for the lack of benefit is that obesity affects aspirin efficacy. The prevalence of obesity in United States skyrocketed from 12\% to $19.8 \%$ between 1991 and $2000(10,11)$, and as an estimated 58\% of world population are projected to be overweight or obese by the year $2030(12,13)$. Thus, understanding the role of obesity in aspirin is paramount.

\section{Aspirin resistance (AR)}

Defined as the occurrence of ASCVD events despite consistent use of aspirin at recommended doses, AR may explain some degree of therapy failure in the primary prevention of heart disease (14-16). Studies have found that diabetes mellitus, female gender, post-acute coronary 
syndrome (ACS) or post-coronary artery bypass grafting, history of stent thrombosis, and obesity are all associated with AR (15,17-22). Obesity confers a three- to fourfold increased risk of secondary ASCVD events and a sixfold higher risk of death $(23,24)$. Obesity affects aspirin metabolism in several ways that reduce its suppression of platelet function (15). Increased platelet reactivity combined with obesity-specific metabolic changes creates a prothrombotic state that warrants treatment. Laboratory studies are attempting to quantify AR by multiple mechanisms, including the suppression of thromboxane B2 (TXB2), assays that detect platelet aggregation in whole blood (e.g., the VerifyNow assay), and flow cytometric analysis for various clusters of differentiation $(15,25,26)$. These studies have found that between $2 \%$ and $57 \%$ patients do not respond to aspirin optimally (15,25-29).

$\mathrm{AR}$ in obesity was first described by Cox et al. in 2006 wherein 71 patients taking enteric coated (EC) aspirin showed treatment failure [relative risk (RR) 2.2; $95 \%$ confidence interval (CI), 1.7-3.0] and incomplete TXB2 inhibition (RR 1.3; 95\% CI, 1.2-1.5) for each $10 \mathrm{~kg}$ increase in bodyweight (30). This study paved the way for subsequent trials evaluating the efficacy of aspirin in obesity and highlights the importance of $\mathrm{AR}$ in this population who may need adjusted aspirin therapy to be better protected from primary ASCVD events.

\section{Disruption of primary hemostasis}

Aspirin is unique in its ability to prevent atherothrombotic events compared to other nonsteroidal anti-inflammatory drugs (31). Aspirin exerts its cardio-protective effects by acting as an antiplatelet drug. During clot formation, arachidonic acid (AA) is converted to TXA2, which then activates platelets by upregulating fibrinogen receptors on platelet surface which allows the primary platelet plug to be formed (29,31). Aspirin, unlike other non-steroidal antiinflammatory drugs, irreversibly acetylates cyclooxygenase-1 (COX-1) thereby decreasing TXA2 levels $(31,32)$. Lower levels of TXA2 do not activate platelets as effectively and thus thrombus formation is impeded $(15,33)$.

\section{Mechanisms of AR in obesity}

Laboratory studies focus on two major approaches to AR: pharmacokinetic and pharmacodynamic. Pharmacodynamic explanations revolve around how obesity creates a prothrombotic state that aspirin is unable to suppress.
Laboratory experiments performed by Bordeaux et al. quantified platelet reactivity by measuring COX-1 pathway metabolites in response to triggers for thrombosis such as collagen, adenosine diphosphate, and AA (19). Results showed that obese individuals had greater baseline platelet reactivity compared to non-obese controls. After low-dose aspirin treatment, platelet function tests still showed greater activation in obese individuals compared to non-obese patients. Higher-dose aspirin, to account for the increased volume of distribution in obese patients, also did not reduce platelet reactivity in obese patients (19). It is worth noting that aspirin only inhibits one of the many pathways of platelet activation and atherothrombosis; therefore, patients may continue to have ASCVD events while taking this medication (8). Obesity is associated with increased concentrations of leptin, a hormone that regulates hunger (15). Studies have showed that leptin increases platelet reactivity and produces a prothrombotic environment (34). This shows that obesity intrinsically affects platelet reactivity and makes it more resistant to aspirin. Further, obesity is associated with higher platelet turnover. Thus, the daily $10-15 \%$ of newly added platelets remain activated despite optimal aspirin effect and can be perceived as $\operatorname{AR}(35,36)$.

Pharmacokinetic explanations focus on reduced concentration of aspirin in the body. A one-dose-fitsall approach to aspirin causes variations in serum aspirin concentrations (37). Among obese patients, aspirin does not reach therapeutic levels in bloodstream and is, therefore, less effective (37). Additionally, aspirin acetylates plasma proteins, such as albumin, hemoglobin, and fibrinogen, which all act as competitive antagonists to COX-1 (37). By non-specifically acetylating these proteins, aspirin's ability to acetylate COX-1 is reduced, thereby lowering platelet reactivity. Aspirin is deacetylated and deactivated by enzymes found in the intestinal epithelium, liver, plasma, and red blood cells (RBCs) (38). Obese patients have higher volumes of plasma and RBCs, which can be associated with elevated concentrations of deacetylating enzymes. Thus, aspirin is more readily deactivated in obese individuals, leading to reduced bioavailability (37). By understanding these mechanisms of AR in obesity, overcoming AR becomes possible.

\section{Overcoming AR}

AR may be overcome by modulating weight, aspirin dose, dosing frequency, and medication formulation. Some studies suggest that weight loss reverses the metabolic 
changes associated with obesity-related AR $(39,40)$. Weight loss was associated with reduced baseline platelet reactivity and improved response to aspirin $(39,40)$. In fact, bariatric surgery has also been associated with improved aspirin responsiveness, which suggests that adipocytes may predispose obese patients to a prothrombotic state (40). Therefore, weight loss can reduce baseline platelet reactivity and decreases AR. This reduction in AR can reverse the increased cardiovascular risks associated with AR.

Most studies evaluating aspirin for cardiovascular benefits have looked at low-dose aspirin because it was believed that aspirin does not display a dose-responsiveness in its antithrombotic effect $(15,41)$. It has come to attention recently that a one-dose-fits-all approach could have led to some biases on its effectiveness (37). When higher doses of aspirin (300 mg, $325 \mathrm{mg}, 500 \mathrm{mg}$ ) were compared to lower doses $(75-100 \mathrm{mg})$ in obese patients, higher doses were found to have lower risk of all cardiovascular events (37). At the same time, higher doses of aspirin were associated with an increased risk of gastrointestinal bleeding $(15,37,41)$. This highlights how aspirin dosage plays a role in AR, and modulating dosage can impact AR.

Bleeding risk, especially gastrointestinal, is the major risk of aspirin, and alternate drug formulations have been tried to reduce this risk. Enteric-coating, has not been shown to decrease gastrointestinal (GI) bleeding (35), is associated with increased $A R$, which highlights the role of drug absorption in AR $(30,35,42)$. A randomized, single-blinded, triple-crossover study analyzed the trends in aspirin nonresponsiveness via TXB2 generation when treated with plain aspirin, EC aspirin and lipid-based formulation of aspirin (PL2200) (42). The study tested 325 $\mathrm{mg}$ aspirin on the forty enrolled patients who had non-insulin dependent diabetes and BMI between 30 and $40 \mathrm{~kg} / \mathrm{m}^{2}$. Aspirin nonresponsiveness was lowest in PL2200 (8.1\%) compared to plain aspirin (15.8\%) and EC aspirin (52.8\%) (42). Another study conducted by Peace $e t$ al. stratified aspirin formulations according to weight for overcoming AR (43). They concluded that patients $<90 \mathrm{~kg}$ respond well to $75 \mathrm{mg}$ of EC aspirin while patients $>90 \mathrm{~kg}$ have better benefit with $75 \mathrm{mg}$ of plain aspirin (43). These studies demonstrate how the formulation of aspirin affects AR.

In addition to higher dose and formulations, frequency of aspirin dosing has also been shown to affect AR $(44,45)$. In a study of 36 patients with coronary artery disease and type 2 diabetes mellitus, the VerifyNow assay determined that greater platelet inhibition was achieved with twice daily low dose aspirin compared to once daily administration (44).
This study did not, however, assess bleeding risk associated with twice-daily dosing.

Finally, and perhaps most obviously, nonadherence to prescribed therapy is a leading cause of AR. A study by Hennekens et al. noted noncompliant patients do not have a reduced risk of myocardial infarction (MI) with aspirin therapy, and use of other nonsteroidal anti-inflammatory drugs combined with aspirin nonadherence actually increases the risk of MI (16). Without good, standardized means of measuring outpatient compliance, this mechanism of AR may be the most difficult to overcome. Overall, physicians should consider dose, formulations, and frequency of dosing before prescribing low-dose aspirin to all patients, and weight loss should be a strong, concomitant recommendation.

\section{Adjunctive therapies to improve AR}

While AR can be overcome, there are adjunctive medicines that can be added to aspirin to reduce cardiovascular risks in high-risk patients. Omega-3-fatty acids inhibit COX-1 pathway and decrease platelet AA and TXA2 synthesis (45). In a randomized controlled trial, 30 patients with coronary artery disease treated with low-dose aspirin (75-162 mg) were found to have AR screened with VerifyNow aspirin assay. The patients were divided to receive either $325 \mathrm{mg}$ daily aspirin or their original low-dose of aspirin plus omega-3-fatty acids. The VerifyNow aspirin assay showed that $80 \%$ of omega-3-fatty acid patient were no longer resistant, and no bleeding events occurred with either group. The study found that omega-3-fatty acids are a good addition to aspirin monotherapy since it reduces platelet reactivity and decreases AR (45).

When aspirin is used in combination with other antiplatelet agents, such as dipyridamole, ticlopidine, intravenous glycoprotein IIb/IIIa antagonists, or P2Y12 agents there is variable reduction in ASCVD events $(46,47)$. Rothwell et al. reports that among individuals weighing at least $70 \mathrm{~kg}$, dual antiplatelet therapy (DAPT) is more effective at reducing stroke in patients with a history of ASCVD events, compared to an isolated regimen of lowdose aspirin alone (37). However, adjunctive medications as part of DAPT were not described. It remains to be determined the effect of adjunctive antiplatelet therapy in a specifically obese population.

A recent meta-analysis evaluated the role of Chinese herbal medicine (CHM) on AR (48). After evaluating 1,460 patients from 18 studies, the main conclusions were that CHM 
could reduce platelet aggregation and would not increase the risk of bleeding, but this is primarily based on trials with short courses (approximately four weeks) of therapy. The applicability outside China and to obese patients remains limited, especially since Chinese patented drugs were also included.

\section{Conclusions}

$\mathrm{AR}$ is an emerging concept of interest as global use of aspirin has increased. Obesity not only increases ASCVD risk but also generally decreases aspirin efficacy, based on pharmacokinetic and pharmacodynamic studies. Obesity creates a prothrombotic state secondary to increased serum leptin levels, reduced serum aspirin concentrations, and greater aspirin inactivation. Despite multiple mechanisms of resistance, there are numerous ways to counteract AR in obese patients. These include modulating aspirin dose based on a patient's weight, increasing dose frequency, using different formulations, and combining other medications with aspirin to increase its effect. It is also important to recommend weight loss and highlight the importance of medication adherence. Ultimately, a one-dose-fits-all aspirin regimen may not be optimal in obese patients and requires tailored therapy to best reduce ASCVD events in this growing population.

\section{Acknowledgments}

None.

\section{Footnote}

Conflicts of Interest: Dr. Cave discloses that he serves on the speaker's bureau for Portola pharmaceuticals. The other authors have no conflicts of interest to declare.

Ethical Statement: The authors are accountable for all aspects of the work in ensuring that questions related to the accuracy or integrity of any part of the work are appropriately investigated and resolved.

\section{References}

1. Arnett DK, Blumenthal RS, Albert MA, et al. 2019 ACC/AHA Guideline on the Primary Prevention of Cardiovascular Disease: A Report of the American College of Cardiology/American Heart Association Task Force on Clinical Practice Guidelines. J Am Coll Cardiol 2019. [Epub ahead of print].

2. Hennekens CH, Dyken ML, Fuster V. Aspirin as a therapeutic agent in cardiovascular disease: a statement for healthcare professionals from the American Heart Association. Circulation 1997;96:2751-3.

3. ASCEND Study Collaborative Group, Bowman L, Mafham M, et al. Effects of Aspirin for Primary Prevention in Persons with Diabetes Mellitus. N Engl J Med 2018;379:1529-39.

4. Gaziano JM, Brotons C, Coppolecchia R, et al. Use of aspirin to reduce risk of initial vascular events in patients at moderate risk of cardiovascular disease (ARRIVE): a randomised, double-blind, placebo-controlled trial. Lancet 2018;392:1036-46.

5. McNeil JJ, Nelson MR, Woods RL, et al. Effect of Aspirin on All-Cause Mortality in the Healthy Elderly. N Engl J Med 2018;379:1519-28.

6. Bibbins-Domingo K, U.S. Preventive Services Task Force. Aspirin Use for the Primary Prevention of Cardiovascular Disease and Colorectal Cancer: U.S. Preventive Services Task Force Recommendation Statement. Ann Intern Med 2016;164:836-45.

7. Hennekens CH, Buring JE, Sandercock P, et al. Aspirin and other antiplatelet agents in the secondary and primary prevention of cardiovascular disease. Circulation 1989;80:749-56.

8. Antithrombotic Trialists' (ATT) Collaboration, Baigent C, Blackwell L, et al. Aspirin in the primary and secondary prevention of vascular disease: collaborative meta-analysis of individual participant data from randomised trials. Lancet 2009;373:1849-60.

9. Flint AJ, Rexrode KM, Hu FB, et al. Body mass index, waist circumference, and risk of coronary heart disease: a prospective study among men and women. Obes Res Clin Pract 2010;4:e171-81.

10. Mokdad AH, Bowman BA, Ford ES, et al. The continuing epidemics of obesity and diabetes in the United States. JAMA 2001;286:1195-200.

11. Mokdad AH, Serdula MK, Dietz WH, et al. The spread of the obesity epidemic in the United States, 1991-1998. JAMA 1999;282:1519-22.

12. Kelly T, Yang W, Chen CS, et al. Global burden of obesity in 2005 and projections to 2030. Int J Obes (Lond) 2008;32:1431-7.

13. Patel JP, Roberts LN, Arya R. Anticoagulating obese patients in the modern era. Br J Haematol 2011;155:137-49. 
14. Szczeklik A, Musiał J, Undas A, et al. Aspirin resistance. J Thromb Haemost 2005;3:1655-62.

15. Grinstein J, Cannon CP. Aspirin resistance: current status and role of tailored therapy. Clin Cardiol 2012;35:673-81.

16. Hennekens CH, Schneider WR, Hebert PR, et al. Hypothesis formulation from subgroup analyses: nonadherence or nonsteroidal anti-inflammatory drug use explains the lack of clinical benefit of aspirin on first myocardial infarction attributed to "aspirin resistance". Am Heart J 2010;159:744-8.

17. Rocca B, Santilli F, Pitocco D, et al. Abstract 12233: Variability in the Recovery Rate of Platelet Cyclooxygenase Activity During Chronic Therapy with Low-Dose Aspirin in Type 2 Diabetes. Circulation 2010;122:A12233.

18. Winocour PD. Platelet turnover in advanced diabetes. Eur J Clin Invest 1994;24 Suppl 1:34-7.

19. Bordeaux BC, Qayyum R, Yanek LR, et al. Effect of obesity on platelet reactivity and response to low-dose aspirin. Prev Cardiol 2010;13:56-62 .

20. Tamminen M, Lassila R, Westerbacka J, et al. Obesity is associated with impaired platelet-inhibitory effect of acetylsalicylic acid in nondiabetic subjects. Int J Obes Relat Metab Disord 2003;27:907-11.

21. Becker DM, Segal J, Vaidya D, et al. Sex differences in platelet reactivity and response to low-dose aspirin therapy. JAMA 2006;295:1420-7.

22. Qayyum R, Becker DM, Yanek LR, et al. Platelet inhibition by aspirin 81 and $325 \mathrm{mg} /$ day in men versus women without clinically apparent cardiovascular disease. Am J Cardiol 2008;101:1359-63.

23. Gum PA, Kottke-Marchant K, Welsh PA, et al. A prospective, blinded determination of the natural history of aspirin resistance among stable patients with cardiovascular disease. J Am Coll Cardiol 2003;41:961-5.

24. Krasopoulos G, Brister SJ, Beattie WS, et al. Aspirin "resistance" and risk of cardiovascular morbidity: systematic review and meta-analysis. BMJ 2008 Jan 26;336:195-8.

25. Kasotakis G, Pipinos II, Lynch TG. Current evidence and clinical implications of aspirin resistance. J Vasc Surg 2009;50:1500-10.

26. Sane DC, McKee SA, Malinin AI, et al. Frequency of aspirin resistance in patients with congestive heart failure treated with antecedent aspirin. Am J Cardiol 2002;90:893-5.

27. Frelinger AL, Furman MI, Linden MD, et al. Residual arachidonic acid-induced platelet activation via an adenosine diphosphate-dependent but cyclooxygenase-1- and cyclooxygenase-2-independent pathway: a 700-patient study of aspirin resistance. Circulation 2006;113:2888-96.

28. Weber AA, Przytulski B, Schanz A, et al. Towards a definition of aspirin resistance: a typological approach. Platelets 2002;13:37-40.

29. Fitzgerald R, Pirmohamed M. Aspirin resistance: effect of clinical, biochemical and genetic factors. Pharmacol Ther 2011;130:213-25.

30. Cox D, Maree AO, Dooley M, et al. Effect of enteric coating on antiplatelet activity of low-dose aspirin in healthy volunteers. Stroke 2006;37:2153-8.

31. Patrono C. The Multifaceted Clinical Readouts of Platelet Inhibition by Low-Dose Aspirin. J Am Coll Cardiol 2015;66:74-85.

32. Patrignani P, Filabozzi P, Patrono C. Selective cumulative inhibition of platelet thromboxane production by low-dose aspirin in healthy subjects. J Clin Invest 1982;69:1366-72.

33. Patrono C, Coller B, Dalen JE, et al. Platelet-active drugs: the relationships among dose, effectiveness, and side effects. Chest 2001;119:39S-63S.

34. Konstantinides S, Schäfer K, Koschnick S, et al. Leptindependent platelet aggregation and arterial thrombosis suggests a mechanism for atherothrombotic disease in obesity. J Clin Invest 2001;108:1533-40.

35. Patrono C, Rocca B. Type 2 Diabetes, Obesity, and Aspirin Responsiveness. J Am Coll Cardiol 2017;69:613-5.

36. Giaretta A, Rocca B, Di Camillo B, et al. In Silico Modeling of the Antiplatelet Pharmacodynamics of Lowdose Aspirin in Health and Disease. Clin Pharmacol Ther 2017;102:823-31.

37. Rothwell PM, Cook NR, Gaziano JM, et al. Effects of aspirin on risks of vascular events and cancer according to bodyweight and dose: analysis of individual patient data from randomised trials. Lancet 2018;392:387-99.

38. Schrör K. Acetylsalicylic Acid. Weinheim: WileyBlackwell, 2009. Available online: http://tahirkhanbooks. synthasite.com/resources/Acetylsalicylic Acid.pdf

39. Davì G, Guagnano MT, Ciabattoni G, et al. Platelet activation in obese women: role of inflammation and oxidant stress. JAMA 2002;288:2008-14.

40. Norgard NB, Monte SV, Fernandez SF, et al. Aspirin responsiveness changes in obese patients following bariatric surgery. Cardiovasc Ther 2017. doi: 10.1111/17555922.12268.

41. Patrono C, Coller B, FitzGerald GA, et al. Platelet-active drugs: the relationships among dose, effectiveness, and side effects: the Seventh ACCP Conference on Antithrombotic and Thrombolytic Therapy. Chest 2004;126:234S-64S. 
42. Bhatt DL, Grosser T, Dong JF, et al. Enteric Coating and Aspirin Nonresponsiveness in Patients with Type 2 Diabetes Mellitus. J Am Coll Cardiol 2017;69:603-12.

43. Peace A, McCall M, Tedesco T, et al. The role of weight and enteric coating on aspirin response in cardiovascular patients. J Thromb Haemost 2010;8:2323-5.

44. Capodanno D, Patel A, Dharmashankar K, et al. Pharmacodynamic effects of different aspirin dosing regimens in type 2 diabetes mellitus patients with coronary artery disease. Circ Cardiovasc Interv 2011;4:180-7.

45. Lev EI, Solodky A, Harel N, et al. Treatment of aspirinresistant patients with omega-3 fatty acids versus aspirin

Cite this article as: Ardeshna D, Khare S, Jagadish PS, Bhattad V, Cave B, Khouzam RN. The dilemma of aspirin resistance in obese patients. Ann Transl Med 2019;7(17):404. doi: 10.21037/ atm.2019.07.52 dose escalation. J Am Coll Cardiol 2010;55:114-21.

46. Antithrombotic Trialists' Collaboration. Collaborative meta-analysis of randomised trials of antiplatelet therapy for prevention of death, myocardial infarction, and stroke in high risk patients. BMJ 2002;324:71-86.

47. Yusuf S, Zhao F, Mehta SR, et al. Effects of clopidogrel in addition to aspirin in patients with acute coronary syndromes without ST-segment elevation. N Engl J Med 2001;345:494-502.

48. Chen H, Shen Z, Chen J, et al. Chinese Herbal Medicine for Aspirin Resistance: A Systematic Review and MetaAnalysis. PLoS One 2016;11:e0154897. 